\title{
Direct Colorimetric Assay for Rapid Detection of Rifampin-Resistant Mycobacterium tuberculosis
}

\author{
Getahun Abate, ${ }^{1,2 *}$ Abraham Aseffa, ${ }^{1}$ Alemayehu Selassie, ${ }^{3}$ Solomon Goshu, ${ }^{3}$ Bekele Fekade, ${ }^{3}$ \\ Dawit WoldeMeskal, ${ }^{1}$ and Håkan Miörner ${ }^{4}$ \\ Armauer Hansen Research Institute ${ }^{1}$ and St. Peter Tuberculosis Specialized Hospital, ${ }^{3}$ Addis Ababa, Ethiopia; Departments of \\ Internal Medicine and Molecular Microbiology, Division of Infectious Diseases and Immunology, Saint Louis University \\ Health Sciences Center, St. Louis, Missouri' ; and Department of Medical Microbiology, Dermatology and Infection, \\ Lund University, Lund, Sweden ${ }^{4}$
}

Received 26 June 2003/Returned for modification 18 September 2003/Accepted 23 October 2003

\begin{abstract}
The colorimetric 3-(4,5-dimethylthiazol-2-yl)-2,5-diphenyl tetrazolium bromide (MTT) assay was standardized for direct detection of rifampin-resistant Mycobacterium tuberculosis in sputum samples. The sensitivity and specificity of the direct MTT assay matched those of the standard indirect susceptibility assay on $7 \mathrm{H10}$ medium, and interpretable results were obtained for $98.5 \%$ of the samples within 2 weeks. Traditional methods of in vitro drug susceptibility testing are time consuming and laborious. Susceptibility tests on clinical isolates require 6 to 9 weeks, and tests conducted directly on smear-positive samples take about 3 weeks (International Union Against Tuberculosis and Lung Disease, The public health service national tuberculosis reference laboratory and the national laboratory network. Minimum requirements, role and operation in a low-income country, Paris, France, 1998, and P. T. Kent and G. P. Kubica, Public health mycobacteriology. A guide for the level III laboratory, Centers for Disease Control and Prevention, Atlanta, Ga., 1985). More-rapid methods are available but are very expensive for routine use under program conditions in countries with high levels of tuberculosis endemicity.
\end{abstract}

A colorimetric assay based on 3-(4,5-dimethylthiazol-2-yl)2,5-diphenyl tetrazolium bromide (MTT) was previously standardized and evaluated using a BACTEC radiometric method as a "gold standard" for indirect detection of rifampin resistance $(1,8)$. MTT is a yellow tetrazolium salt that is converted into blue formazan by dehydrogenases of live cells (7). The amount of blue or purple formazan formation is proportional to the number of live mycobacteria in a sample (8). The results of the MTT assay matched fully with the results obtained using the BACTEC method (1).

This study was conducted with the objectives of standardizing the MTT assay for the detection of rifampin resistance directly on smear-positive sputum samples and evaluating the assay against the traditional method as the gold standard.

Standardization and evaluation. Sputum samples from 16 new smear-positive cases of pulmonary tuberculosis were used to standardize the assay. Sputum samples from 74 smear-positive retreatment cases were pelleted after digestion and decontamination with $4 \% \mathrm{NaOH}(5)$. The pellets were neutralized and resuspended in $3 \mathrm{ml}$ of sterile $7 \mathrm{H} 9$ broth each. An aliquot of $500 \mu \mathrm{l}$ was added to tubes containing $3 \mathrm{ml}$ of $7 \mathrm{H} 9$ Middlebrook broth supplemented with $10 \%$ oleic-acid-albumin-dextrose-catalase, glycerol $(0.05 \%)$, and PANTA (Becton Dickinson, Paramus, N.J.) (75 $\mu \mathrm{l})$. Rifampin (Sigma) at a final concentration of $2 \mu \mathrm{g} / \mathrm{ml}$ was added to some of the tubes. The experimental approach is summarized in Fig. 1. All tubes were incubated at $37^{\circ} \mathrm{C}$ until the day of the MTT assay.

\footnotetext{
* Corresponding author. Mailing address: Division of Infectious Diseases \& Immunology, Departments of Internal Medicine \& Molecular Microbiology, Saint Louis University Health Sciences Center, 3635 Vista Ave., FDT-8N, St. Louis, MO 63110. Phone: (314) 5778690. Fax: (314) 771-3816. E-mail: abateg@slu.edu.
}

MTT assay. The MTT assay was done each week for 3 weeks. Contamination was checked by growing subcultures on nutrient agar medium. Each week, an MTT assay was done using one bacterial control tube with no rifampin and another tube with bacteria and rifampin. The assay was done as described previously (1). Relative optical density (OD) unit (RODU) values were calculated for each sample by dividing the OD value of the rifampin-containing tube by the OD value of the drug-free control. Resistance was defined as RODU $>$ 0.5 , and susceptibility was defined as RODU $<0.2$. RODU values obtained each week for samples containing susceptible isolates were compared (using the Mann-Whitney U test) with those of samples containing resistant isolates. The lowest OD value which was considered indicative of growth was determined by growing a subculture with an aliquot of vortexed broth medium every week before the MTT assay. The lowest OD value with a positive culture result was 0.10 ; therefore, the results were considered interpretable when the OD value of the control was $\geq 0.1$.

Standard sensitivity testing. Standard biochemical tests were used to identify all isolates as Mycobacterium tuberculosis (5). A proportion method (5) using Middlebrook 7H10 medium was used as a reference method for rifampin susceptibility testing. Reference M. tuberculosis strains ATCC 35836 (rifampin susceptible) and ATCC 35838 (rifampin resistant) were used as controls.

Among the 74 samples used to evaluate the MTT assay, 5 (6.8\%) were excluded, 3 because the OD values of control tubes remained below 0.1 and 2 because there was no growth on Löwenstein-Jensen medium. Of the remaining 69 samples, $5(7.2 \%)$ were contaminated; however, for each of the five samples there was at least one noncontaminated interpretable 


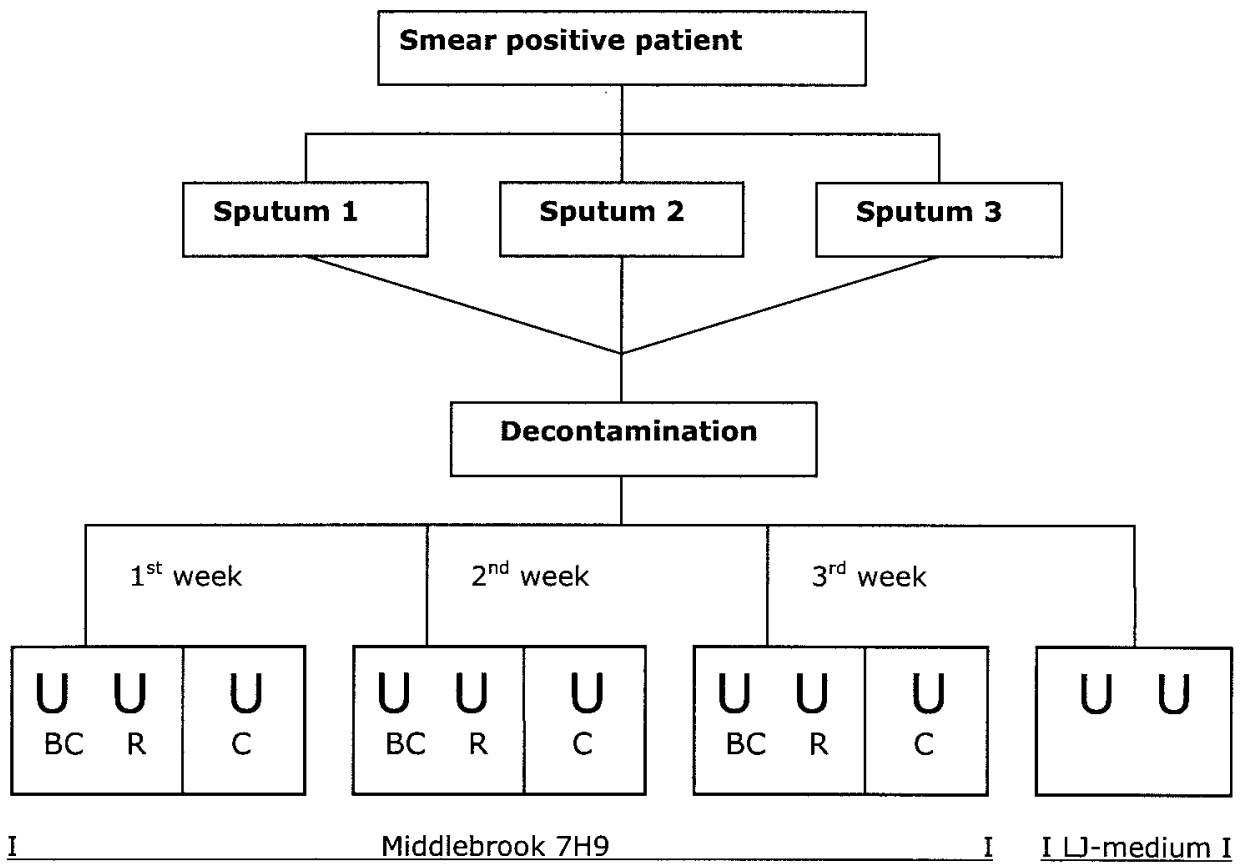

FIG. 1. Flow chart of MTT assay. Abbreviations: BC, bacterial control without rifampin; R, tube with $2 \mu \mathrm{g}$ of rifampin/ml; C, control without bacteria. LJ, Löwenstein-Jensen.

result among the results of the three sets of experiments prepared for the 3 weeks. The contamination rates of 18 samples tested in the presence of PANTA (Becton Dickinson) and in the presence of a much cheaper but similar antibiotic cocktail prepared in-house were the same. There was no contamination in the presence of Löwenstein-Jensen medium.

Table 1 shows the number of interpretable results obtained in each week. In the first week, 43 of $68(63 \%)$ of the samples gave interpretable results; the number of samples with interpretable results grew in the second $(98.5 \%)$ and third $(100 \%)$ weeks. The MTT assay identified 8 of $69(11.6 \%)$ samples as containing rifampin-resistant M. tuberculosis and 61 of 69 $(88.4 \%)$ as containing rifampin-susceptible $M$. tuberculosis. The susceptibility (sensitivity and specificity) results obtained

TABLE 1. Contamination rate and time of interpretation of a direct MTT assay for detection of rifampin-resistant M. tuberculosis $^{a}$

\begin{tabular}{|c|c|c|c|c|c|c|}
\hline \multirow{3}{*}{$\begin{array}{l}\text { Assay } \\
\text { week }\end{array}$} & \multirow{3}{*}{$\begin{array}{c}\text { No. of } \\
\text { contaminated } \\
\text { samples/total no. of } \\
\text { samples tested }(\%)\end{array}$} & \multirow{3}{*}{$\begin{array}{c}\text { No. of } \\
\text { interpretable } \\
\text { samples/total no. of } \\
\text { samples tested }(\%)\end{array}$} & \multicolumn{4}{|c|}{$\begin{array}{c}\text { No. of samples } \\
\text { giving the indicated } \\
\text { assay result }\end{array}$} \\
\hline & & & \multicolumn{2}{|c|}{ MTT } & \multicolumn{2}{|c|}{$\begin{array}{c}7 \mathrm{H} 10 \\
\text { (indirect) }\end{array}$} \\
\hline & & & $\mathrm{S}$ & $\mathrm{R}$ & $\mathrm{S}$ & $\mathrm{R}$ \\
\hline 1 & $1 / 69(1.4)$ & $43 / 68(63)$ & 37 & 6 & & \\
\hline 2 & 4/69 (5.8) & $64 / 65(98.5)$ & 56 & 8 & & \\
\hline 3 & $3 / 69(4.3)$ & $66 / 66(100)$ & 58 & 8 & & \\
\hline Total & $8 / 207$ (3.9) & $69 / 69(100)$ & 61 & 8 & 61 & 8 \\
\hline
\end{tabular}

${ }^{a}$ Samples were inoculated in duplicates into drug-free broth medium. The change in OD was measured with the MTT assay (1) at 1 and 2 weeks after inoculation.

${ }^{b} \mathrm{~S}$, susceptible; R, resistant. with MTT concurred fully with findings obtained using the standard assay on 7H10 agar medium. Figure 2 shows that the RODU values of samples containing susceptible bacteria remained below 0.2 in all weeks of experiments and that the RODU values of samples containing resistant bacteria were above 0.5 . The differences in the RODU values of samples

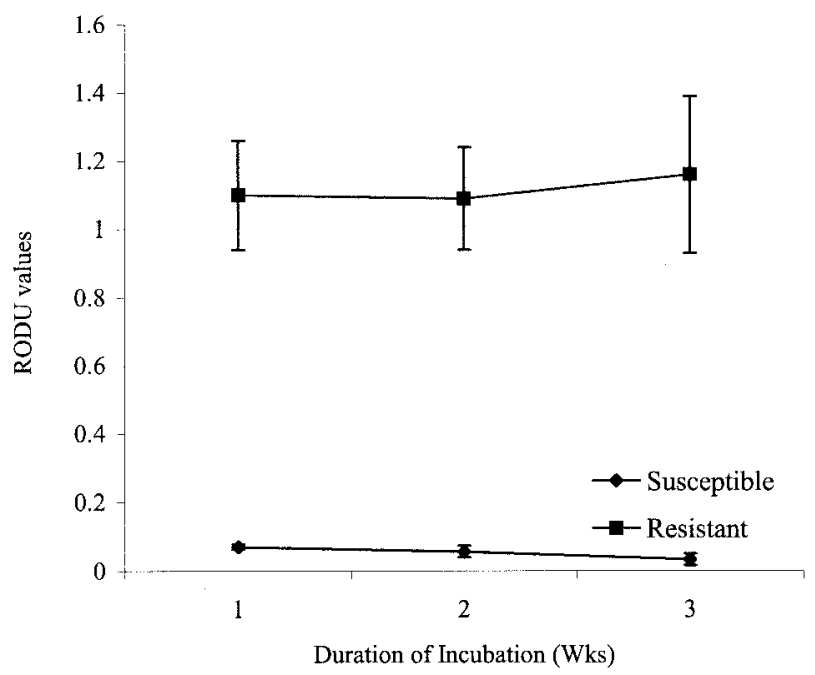

FIG. 2. Growth patterns of rifampin-susceptible $(n=37$ samples in the first week, 56 in the second week, and 58 in the third week) and rifampin-resistant $(n=6$ in the first week and 8 in the subsequent weeks) strains of $M$. tuberculosis as reflected by RODU values (means \pm standard errors) $\left[\left(\mathrm{RODU}=\mathrm{OD}_{570}\right.\right.$ of rifampin-containing medi$\mathrm{um}) /\left(\mathrm{OD}_{570}\right.$ of drug-free medium $\left.)\right]$. The difference in RODU values in each week was statistically significant $(P<0.01$ [Mann-Whitney test]). 
containing susceptible and resistant isolates were statistically significant for each week $(P<0.01)$.

Our findings show that a direct assay based on a tetrazolium salt significantly reduces the time required to obtain reliable susceptibility results. The standard direct methods of drug sensitivity testing on solid medium take 3 to 4 weeks $(5,6)$, and with these conventional methods, there is in addition a need to prepare appropriate dilutions of a specimen as determined on the basis of smear grading (5). Our application of a direct MTT assay is not dependent on smear grading and shortens the turnaround time. Other direct rapid methods (such as the BACTEC 460 system and the mycobacterial growth indicator tube system) have a turnaround time ranging from 9 to 12 days $(3,6)$. However, they are very expensive for routine use in most countries in which tuberculosis is endemic.

Rifampin resistance is a strong predictor of the presence of multidrug-resistant tuberculosis (2). Therefore, the results of our study focusing on the direct detection of rifampin-resistant M. tuberculosis indicate the potential of this simple and inexpensive assay for control programs in countries with high levels of tuberculosis endemicity. The same assay could theoretically be used to rapidly screen for resistance to other antituberculosis drugs. Our preliminary findings indicate that the same assay could be used for reliable and rapid detection of isoniazid resistance. This new method should be evaluated under program conditions in a region with a high level of tuberculosis endemicity and optimized for program use.
This project was supported by a grant from the UNDP/WORLD BANK/WHO Special Programme for Research and Training in Tropical Diseases (TDR).

\section{REFERENCES}

1. Abate, G., R. N. Mshana, and H. Miörner. 1998. Evaluation of a colorimetric assay based on 3-(4,5-dimethyl-2-yl)-2,5-diphenyl tetrazolium bromide for rapid detection of rifampin resistance in Mycobacterium tuberculosis. Int. J. Tuberc. Lung Dis. 2:1011-1016.

2. Abate, G., H. Miörner, O. Ahmed, and S. E. Hoffner. 1998. Drug resistance in Mycobacterium tuberculosis strains isolated from re-treatment cases of pulmonary tuberculosis in Ethiopia: susceptibility to first-line and alternative drugs. Int. J. Tuberc. Lung Dis. 2:580-584.

3. Goloubeva, V., M. Lecocq, P. Lassowsky, F. Matthys, F. Portaels, and I. Bastian. 2001. Evaluation of mycobacteria growth indicator tube for direct and indirect drug susceptibility testing of Mycobacterium tuberculosis from respiratory specimens in a Siberian prison hospital. J. Clin. Microbiol. 39: 1501-1505.

4. International Union Against Tuberculosis and Lung Disease. 1998. The public health service national tuberculosis reference laboratory and the national laboratory network. Minimum requirements, role and operation in a lowincome country. International Union Against Tuberculosis and Lung Disease, Paris, France.

5. Kent, P. T., and G. P. Kubica. 1985. Public health mycobacteriology. A guide for the level III laboratory. Centers for Disease Control and Prevention, Atlanta, Ga.

6. Libonati, J. P., C. E. Stager, J. R. Davis, and S. H. Siddiqi. 1988. Direct antimicrobial drug susceptibility testing of Mycobacterium tuberculosis by the radiometric method. Diagn. Microbiol. Infect. Dis. 10:41-48.

7. Mossman, T. 1983. Rapid colorimetric assay for cellular growth and survival: application to proliferation and cytotoxicity assays. J. Immunol. Methods 65:55-63.

8. Mshana, R. N., G. Tadesse, G. Abate, and H. Miörner. 1998. Use of 3-(4,5dimethyl-2-yl)-2,5-diphenyl tetrazolium bromide for rapid detection of rifampin-resistant Mycobacterium tuberculosis. J. Clin. Microbiol. 36:1214-1219. 